Methods 37 consecutive patients with CTO lesion in LAD coronary artery who underwent PCI as well as SPECT/CTA fusion imaging were divided into the following three groups based on the myocardial perfusion index before PCI: (A) no severe cardiac perfusion defects $(n=4)$; (B) reversible cardiac perfusion defects $(n=18) ;(C)$ irreversible cardiac perfusion defects $(n=15)$.

Results Overall successful rate of recanalisation for CTO was $75.7 \%$ (28/37). No statistical difference of perfusion abnormality was observed 6 months after PCI in group A. In group B, SPECT/CTA fusion imaging demonstrated that cardiac perfusion abnormality was significantly decreased 6 months after PCI $(21 \pm 7.9 \%$ vs $28 \pm 9.8 \%$, $\mathrm{p}<0.05)$. Left ventricular ejection fraction (LVEF) significantly enhanced as evaluated by echocardiography $(51 \pm 8.3 \%$ vs $43 \pm 6.2 \%$, $\mathrm{p}<0.05)$ as well as SPECT $(50 \pm 7.7 \%$ vs $45 \pm 8.5 \%, \mathrm{p}<0.05)$ compared with baseline. Quality of life improved as evidenced by 6 -min walk distance (6MWD) $(426.4 \pm 33.8 \mathrm{~m}$ vs $347.3 \pm 24.6 \mathrm{~m}$, $\mathrm{p}<0.05)$ and angina pectoris score index $(60.8 \pm 13.5$ vs $53.7 \pm 11.2$, $\mathrm{p}<0.05)$. Moreover, patients in group $\mathrm{C}$ also benefited from PCI therapy: a decrease in cardiac perfusion abnormality, an increase in LVEF and an improvement in quality of life.

Conclusion PCI exerts long-term functional and clinical benefits in patients with CTO lesion in LAD coronary artery, particularly in patients with reversible cardiac perfusion defects. SPECT/CTA fusion imaging may serve as a gatekeeper to evaluate the outcomes of patients with CTO lesion in LAD coronary artery.

\section{Q0470 ULTRASOUND GUIDED THROMBIN INJECTION FOR THE TREATMENT OF IATROGENIC POSTCATHETERISATION PSEUDOANEURYSMS IN 76 CASES}

doi:10.1136/hrt.2010.208967.470

${ }^{1}$ Guo Jincheng, ${ }^{2}$ Ma Changsheng, ${ }^{1} \mathrm{Xu}$ Min, ${ }^{1}$ Wang Guozhong, 'Zhang Zhenghai, ${ }^{1}$ Zhang Linxin. ${ }^{1}$ Luhe Hospital; ${ }^{2}$ An Zhen Hospital

Ultrasound-Guided Thrombin Injection For The Treatment Of Iatrogenic Post-Catheterisation Pseudoaneurysms In 76 Cases Objective The purpose of this study was to evaluate the safety and efficacy of ultrasound-guided thrombin injection (UGTI) for the treatment of iatrogenic post-catheterisation pseudoaneurysms (PSAs).

Methods A total of 76 patients (36 men, 40 women, $63.4 \pm 10.8$ years) with iatrogenic PSAs were treated by UGTI

Results The mean diameter of the aneurysms was $(3.01 \pm 1.27) \times$ $(1.65 \pm 0.67) \mathrm{cm}, 93.4 \%(71 / 76)$ of the patients were under antiplatelet therapy with aspirin or clopidogrel or both, and additional low molecular weight heparin. The mean dose of bovine thrombin was $619 \pm 259(150 \sim 1400) \mathrm{u}$, single injection was primary successful in 69 patients, of which thrombus formation occurred in 1 patient in the superficial femoral artery after successful closure of the PSA, surgical embolectomy was performed. A second injection was required in 2 of the remaining 7 patients. 5 patients were treated by ultrasoundguided compression because of incomplete thrombosis after UGTI. 1 patient had acute allergy after 2 min of thrombin injection which was resolved by antiallergic therapy. The total success rate was $98.7 \%$ (75/76). reperfusion was detected in 4 patients within $72 \mathrm{~h}$ followup, recurrence rate of UGTI for PSAs was 5.3\% (4/75), 3 patients were successfully managed by a second thrombin injection and another was treated with ultrasuound-guidance compression, there is no recurrence at 30 days clinical follow up. ultrasound follow-up. At the 2 months were performed in 15 patients. The size of PSAs were significantly reduced from $(2.90 \pm 1.17),(1.47 \pm 0.54) \mathrm{cm}$ to $(0.94 \pm 0.72)^{\prime}(0.44 \pm 0.35) \mathrm{cm}(\mathrm{p}<0.001)$.

Conclusion UGTI is a safe, rapid, well-tolerated and effective noninvasive method for the treatment of iatrogenic PSAs and should be considered as first-line therapy.

\section{e0471 CLINICAL INVESTIGATION OF TRANSRADIAL APPROACH FOR EMERGENT PERCUTANEOUS CORONARY INTERVENTION IN PATIENTS WITH ACUTE MYOCARDIAL INFARCTION}

doi:10.1136/hrt.2010.208967.471

Xuguang Qin, Chengjie Gong, Weiguo Xiong, Chunpeng Lu. Department of Cardiology, First Affiliated Hospital of Tsinghua University. Beijing, China

Objective To evaluate the safety and efficacy of transradial approach for emergent percutaneous coronary intervention in patients with acute myocardial infarction.

Methods We analysed data from our single-center registry on 560 consecutive patients between January 2001 and October 2009. All the patients were respectively randomised to transradial group $(n=260)$ and trans-femoral group $(n=300)$. A dedicated doctor was appointed to collect such indicators as follows: puncture time, CAG time, PCI time, $\mathrm{x}$-ray exposure time, complication rates associated with puncture such as puncture site bleeding, haematoma, pseudoaneurysm, and the major adverse cardiac events.

Results 1 . There were no significant differences in the baseline characteristics and angiographic findings between two groups. 2. There was no significant differences in CAG time $(8 \pm 2.4 \mathrm{~min}$ vs $7.6 \pm 2.0 \mathrm{~min})$, PCI time $(30 \pm 4.8 \mathrm{~min}$ vs $28.6 \pm 4.4 \mathrm{~min})$, and $\mathrm{x}$-ray exposure time ( $4.6 \pm 1.4 \mathrm{~min}$ vs $4.4 \pm 1.3 \mathrm{~min})$ between two groups. 3 . The complication rates of TRA was $2.32 \%(6 / 260)$, compared to $6.0 \%(18 / 300)$ in the control group $(\mathrm{p}<0.05)$.

Conclusion Transradial approach for emergent percutaneous coronary intervention in patients with acute myocardial infarction is safe and efficacy, and it is suggested that the transradial approach should be used in patients with acute myocardial infarction.

\section{e0472 TWO-YEAR CLINICAL EFFICACY OF SIROLIMUS-VERSUS PACLITAXEL-VERSUS ZOTAROLIMUS-ELUTING STENTS IN DIABETIC PATIENTS}

doi:10.1136/hrt.2010.208967.472

${ }^{1}$ Kang-Yin Chen, ${ }^{2}$ Seung-Woon Rha, ${ }^{3}$ Yong-Jian Li, ${ }^{1}$ Guang-Ping Li, ${ }^{1} \mathrm{Ji}-$ Young Park, ${ }^{1}$ Sureshkumar Ramasamy, ${ }^{1}$ Kanhaiya Poddar, ${ }^{1}$ Byoung Geol Choi, ${ }^{1} Y u n$ Kyung Kim, ${ }^{1} \mathrm{Jin} \mathrm{Oh} \mathrm{Na},{ }^{1}$ Cheol Ung Choi, ${ }^{1}$ Hong Euy Lim, ${ }^{1} \mathrm{Jin}$ Won Kim, ${ }^{1}$ Eung Ju Kim, ${ }^{1}$ Chang Gyu Park, ${ }^{1}$ Hong Seog Seo, Dong Joo Oh. ${ }^{1}$ Korea University Guro Hospital; ${ }^{2}$ Tianjin Nankai Hospital; ${ }^{3}$ The Second Hospital Of Tianjin Medical University

Background Drug-eluting stents (DESs) have drastically improved the angiographic and clinical outcomes of percutaneous coronary intervention (PCI) in patients (pts) with diabetes mellitus. However, little has been known whether the different types of DESs have similar efficacy in Asian diabetic pts.

Methods A total of 305 diabetic pts who underwent PCI with Sirolimus (SES group; Cypher, $\mathrm{n}=102$ pts, 247 lesions), Paclitaxel (PES group; Taxus, $\mathrm{n}=138$ pts, 414 lesions) or Zotarolimus (ZES group; Endeavour, $n=65$ pts, 138 lesions)-eluting stents were enrolled. Angiographic outcomes at 6 months and cumulative clinical outcomes up to 2 years were compared among these 3 groups. Results These 3 groups had similar baseline clinical and procedural characteristics except that SES group had longer stent length and PES group had smaller stent diameter as compared with other groups. Six-month angiographic outcomes showed that SES group had less binary restenosis, lower restenosis percent, and late loss as compared with the other 2 groups. Major clinical outcomes were similar among the 3 groups up to 2 years except a trend towards lower incidence of TVR in SES group as compared with the other 2 groups. ZES group had 1 acute, 1 subacute, and 1 late stent thrombosis (ST), while the other 2 groups didn't have ST throughout the follow-up period (Table). 